\title{
Use of the 1 tuberculin unit (TU) Mantoux test in the assessment of tuberculous infection in children following neonatal BCG vaccination
}

\author{
E W Hoskyns, H Simpson, P Monk
}

\begin{abstract}
Background - BCG vaccination alters the response to tuberculin testing and influences the potential validity of the Heaf test in the diagnosis of tuberculosis. This study used a purified protein derivative 1 tuberculin unit (TU) Mantoux test with a cut off of $5 \mathrm{~mm}$ induration as an indicator of tuberculous infection in high risk children to determine whether this would distinguish infection from previous neonatal BCG vaccination.
\end{abstract}

Methods - Children at high risk of tuberculosis on chest radiography, Heaf test, or contact history who had been screened in the contact tracing clinic and referred for further assessment were included in the study. After clinical examination, chest radiography, and Mantoux testing they were assigned to three groups (tuberculous disease, chemoprophylaxis, or no treatment) and followed up for 6-24 months in the outpatient clinic and subsequently by postal questionnaire.

Results - Comparison of the Heaf and Mantoux tests showed a difference in the results with $82 \%$ of cases positive by the Heaf test and $59 \%$ positive by the Mantoux test. Using the Mantoux test result in combination with clinical and radiographic findings 194 children were allocated to the three groups as follows: primary tuberculosis (5), chemoprophylaxis (101), no treatment (88). During follow up for a mean (range) time of 46 (11-102) months four additional cases received treatment for primary tuberculosis, two in the chemoprophylaxis group and two in the untreated group.

Conclusions - The use of the 1 TU Mantoux test after neonatal BCG vaccination reduced the number of children receiving treatment from 129 to 93 - that is, by $36 \%$. Although the numbers are small, there was no increase in the later development of tuberculosis.

(Thorax 1994;49:1006-1009)

The natural history of tuberculosis begins with infection which may, after a period of time, progress to clinical disease. Many factors determine the progression from infection to disease, one of which is the age at which infection occurs. ${ }^{1}$ Infected younger children are much more likely to progress to primary and haematogenous disease. ${ }^{1}$ Infection is diagnosed by tuberculin testing, but previous BCG vaccination alters the tuberculin sensitivity. ${ }^{23}$ In the UK it is recommended that a grade 2 or above Heaf test is considered positive,${ }^{4}$ but if there has been a previous BCG vaccination grade 3 or above should be used. ${ }^{5}$ There is little evidence to support the validity of the latter cut off.

In many districts in the UK children born to groups at high risk of tuberculosis are routinely given BCG vaccination at birth. As by definition they have a high incidence of the disease, they make up a large part of the referrals to contact tracing clinics and it is possible that many children are being treated for a response to the BCG vaccination rather than infection with tuberculosis.

The main aim of this study was to investigate prospectively the use of a 1 tuberculin unit (TU) Mantoux test as an indicator of infection in young tuberculosis contacts. Induration of $5 \mathrm{~mm}$ was taken as the cut off point to distinguish infection from neonatal BCG vaccination. A subsidiary aim was to compare the results of Heaf tests carried out in the referral clinics with those of the 1 TU Mantoux test routinely carried out in the follow up paediatric outpatient setting.

\section{Methods}

REFERRALS

All referrals from the tuberculosis contact clinic to the paediatric chest clinic for the eight years from 1984 to 1991 inclusive were included in the study. Children were referred if one or more of the following were present: (1) clinical and/ or radiological evidence of tuberculosis; (2) grade 3 or 4 Heaf test; (3) Heaf conversion; (4) perceived high risk irrespective of clinical and Heaf test findings. Age, infectivity of source, and degree of contact were the main factors considered.

\section{ASSESSMENT}

The children were assessed clinically and by chest radiography. Tuberculin sensitivity was assessed with purified protein derivative (PPD). Patients who had had previous BCG vaccination as reported by the parents were given an intradermal injection of $0 \cdot 1 \mathrm{ml}$ of 1 in 10000 strength (1 TU); those who had not been previously vaccinated received 1 in 1000 strength (10 TU). The results read after 48-72 hours, in combination with the clinical and radiographic findings, allowed children to be assigned to three treatment groups. 
(1) Primary tuberculosis: all these children had either an abnormal chest radiograph with an appropriate clinical history or biopsy evidence of tuberculosis. Cases were treated with a nine month course of isoniazid 5-10 mg/ $\mathrm{kg} /$ day and rifampicin $10-20 \mathrm{mg} / \mathrm{kg} /$ day. Latterly several children were also treated with a third drug (ethambutol, $15-25 \mathrm{mg} / \mathrm{kg} / \mathrm{day}$ or pyrazinamide, $30-40 \mathrm{mg} / \mathrm{kg} /$ day) for an initial two month period.

(2) Children with a positive Mantoux test and a normal chest radiograph were given chemoprophylaxis with six months isoniazid and rifampicin. A few children with a negative Mantoux test and normal chest radiograph were treated on the basis of a high perceived risk.

(3) Most children who were symptom-free with a normal chest radiograph and a negative Mantoux, and who were apparently healthy, were not treated but followed up.

\section{FOLLOW UP}

Children were followed up whether or not they received treatment or chemoprophylaxis. The initial plan to follow up children six monthly for two years was not always achieved. It seemed important, therefore, to review as many as possible after a longer period to determine as far as possible the outcome for the group as a whole. Information was obtained from a comprehensive review of the medical records of all children referred from 1984 to 1991 inclusive. To review missing children who may have moved out of the area, or presented later to adult services (with separate records), or who had received further treatment in community or paediatric outpatient settings, a postal questionnaire was also sent to each case, having obtained current addresses and names of the general practitioner from the FHSA register of Leicestershire Health Authority. This one page questionnaire enquired about further problems with tuberculosis and treatment prescribed. Non-responders were circulated with a second questionnaire. If there was still no response general practitioner records were perused.

\section{SUBJECTS}

Two hundred and four children were referred to the paediatric chest clinic; 194 (95\%) of their records were available for review. The age distribution of these children is shown in the figure. One hundred and eighty $(92 \%)$ were ethnic Asians and 171 of these (95\%) had a BCG vaccination in the neonatal period, $88 \%$ in the UK. Locally this had been given by intradermal injection of $0.05 \mathrm{ml} \mathrm{BCG}$ vaccine (Evans Medical Ltd) equivalent to a dose of $4 \times 10^{5}-1.3 \times 10^{6}$ colony forming units. The parental history of BCG vaccination was not verified routinely except by observation of a BCG scar. Four children were vaccinated outside the neonatal period and in seven cases (three with an apparent BCG scar) there was uncertainty about BCG status.

\section{Results}

The number of children in the different referral groups is given in table 1 . Of the 194 children for whom records were available 185 (95\%) received a Heaf test. Nine were of grade 1 or less, 28 of grade 2,77 of grade 3 , and 71 of grade 4 - that is, $148(80 \%)$ were Heaf grade 3 or more.

Of the 194 children 182 (94\%) had a 1 TU Mantoux test. Seventy six had induration of $<5 \mathrm{~mm}, 29$ of $5-9 \mathrm{~mm}, 44$ of $10-14 \mathrm{~mm}$, and 33 of $15 \mathrm{~mm}$ or more - that is, $106(58 \%)$ were positive to $>5 \mathrm{~mm}$ induration.

Heaf testing always preceded Mantoux testing by a median time of two months (range two weeks to 10 months) for the 173 patients who had both tests. Table 1 gives the details of the total numbers by race who had both tuberculin tests and neonatal BCG vaccination.

Comparison of the Heaf test with the $1 \mathrm{TU}$ Mantoux test for all those who had had a neonatal BCG vaccination is given in table 2 . There was a significant but weak correlation between the two tests $(r=0.399)$ with significantly more positive cases by Heaf than by Mantoux testing.

Six patients who were vaccinated with BCG

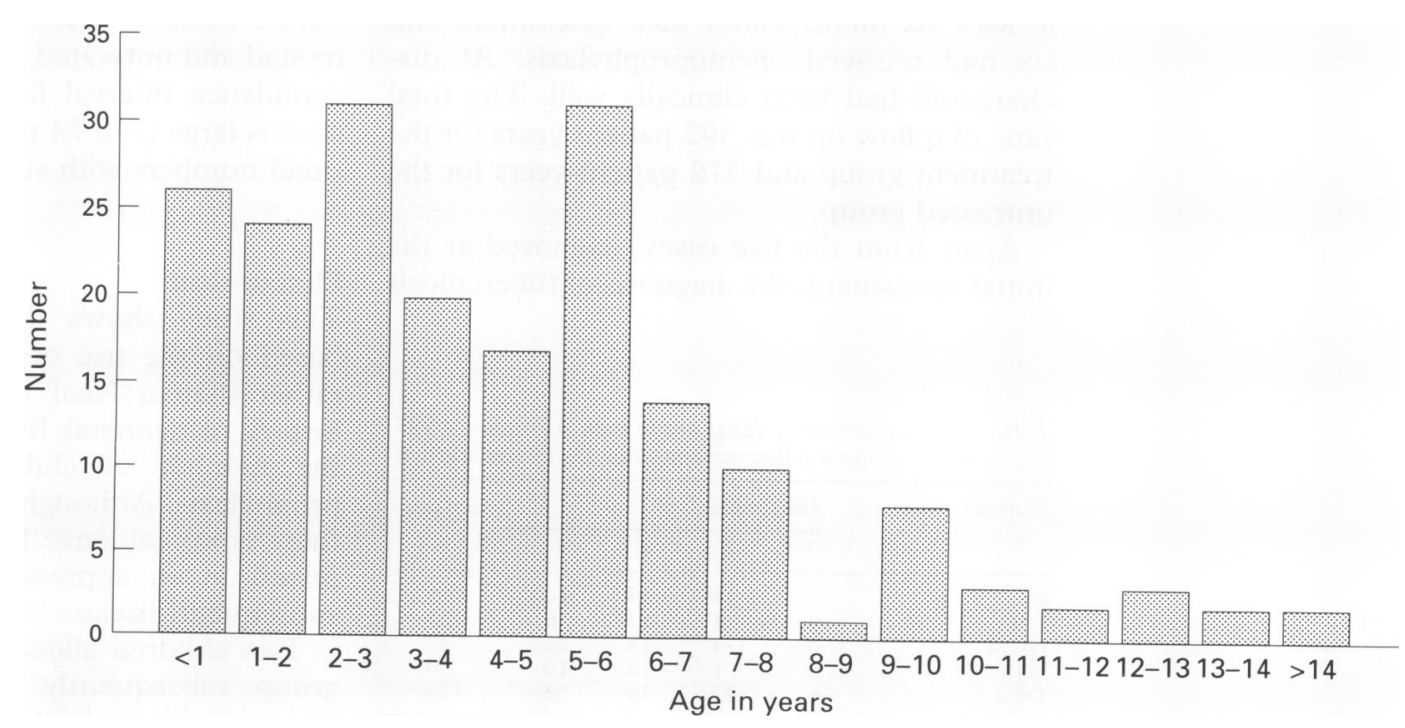


Table 1 Breakdown of numbers for each of the referral groups by tuberculin testing, BCG vaccination and race

\begin{tabular}{|c|c|c|c|c|}
\hline & Contacts & Immigrants & Symptoms & Total \\
\hline $\begin{array}{l}\text { Total (all races) } \\
\% \text { with Heaf grade } \geqslant 3 \\
\% \text { with Mantoux induration } \geqslant 5 \mathrm{~mm}\end{array}$ & $\begin{array}{r}149 \\
82 \\
58\end{array}$ & $\begin{array}{l}38 \\
82 \\
62\end{array}$ & $\begin{array}{r}7 \\
33 \\
33\end{array}$ & $\begin{array}{l}194 \\
185 \\
182\end{array}$ \\
\hline $\begin{array}{l}\text { Asians* } \\
\text { Total } \\
+ \text { neonatal BCG } \\
\text { + BCG and Heaf and Mantoux }\end{array}$ & $\begin{array}{l}139 \\
132 \\
118\end{array}$ & $\begin{array}{l}35 \\
33 \\
32\end{array}$ & $\begin{array}{l}6 \\
6 \\
5\end{array}$ & $\begin{array}{l}180 \\
171 \\
155\end{array}$ \\
\hline $\begin{array}{l}\text { White population } \\
\text { Total } \\
\text { + neonatal BCG } \\
+ \text { BCG and Heaf and Mantoux }\end{array}$ & $\begin{array}{r}10 \\
3 \\
2\end{array}$ & $\begin{array}{l}3 \\
1 \\
1\end{array}$ & $\begin{array}{l}1 \\
0 \\
0\end{array}$ & $\begin{array}{r}14 \\
4 \\
3\end{array}$ \\
\hline
\end{tabular}

* Includes one child of mixed racial origins.

in the neonatal period had a negative Mantoux $1 \mathrm{TU}$ result; on further testing with a Mantoux $10 \mathrm{TU}$ test three were positive.

Among the referrals there were 36 who had had repeat Heaf tests and had shown a Heaf conversion - that is, increase of at least two grades. Seventeen had a negative response to the 1 TU Mantoux test.

\section{TREATMENT AND FOLLOW UP}

Based on the clinical and Mantoux test findings 101 children $(52 \%)$ were given chemoprophylaxis and $88(45 \%)$ no treatment. The remaining five were treated for primary tuberculosis. Six children were not treated despite a positive Mantoux test result and 11 were treated after a negative Mantoux test result. In the latter group three had Heaf tests of grade 2 or less, two of grade 3, and the remainder of grade 4 . The decision to treat was influenced by clinical factors including the age of the child and the degree of contact.

One hundred and eighty four cases were traced in December 1992 giving a median time elapsed since assessment of 46 months. Of these, $76 \%$ responded to postal questionnaire and $19 \%$ were traced via their general practitioners. The 10 untraced children comprised seven who had moved from the area (three out of the country) and three where the identified general practice did not hold the record and could not supply details. These 10 children had been followed in the outpatient clinic for at least six months after their assessment and six had received chemoprophylaxis. At discharge all had been clinically well. The total time of follow up was 392 patient years for the treatment group and 372 patient years for the untreated group.

Apart from the five cases diagnosed at the initial assessment, the diagnosis of tuberculosis

Table 2 Comparison of Heaf grade with Mantoux $1 T U$ test result in children following neonatal BCG vaccination

\begin{tabular}{llrlrl}
\hline Mantoux $(\mathrm{mm})$ & \multicolumn{3}{l}{ Heaf grade } & \multirow{2}{*}{ Total } \\
\cline { 2 - 5 } & $0-1$ & \multicolumn{1}{l}{2} & 3 & \multicolumn{1}{c}{4} & \\
\hline$<5$ & 6 & 14 & 35 & 10 & 65 \\
$5-9$ & 0 & 4 & 13 & 6 & 23 \\
$10-14$ & 0 & 4 & 15 & 21 & 40 \\
$\geqslant 15$ & 0 & 1 & 13 & 16 & 30 \\
Total & 6 & 23 & 76 & 53 & 158 \\
\hline
\end{tabular}

McNemar $\mathrm{p}<0.05$

Spearman rank coefficient $=0 \cdot 39$. was made in four children following their allocation to chemoprophylaxis or no treatment. Two were from the chemoprophylaxis group and two from the non-treated group. No new cases were identified as a result of the questionnaire.

The two children who were not treated initially were a sibling pair who were recent immigrants from the Indian subcontinent. They were 6.5 and 2.5 years old and had no history of contact with tuberculosis. Both had Heaf grade 3 and borderline Mantoux results $(5 \mathrm{~mm})$. Because of the lack of contact history they were not treated. At follow up five months later both were symptom-free but had developed hilar lymphadenopathy on the chest radiograph which responded to antituberculous chemotherapy. They remained asymptomatic throughout.

The two children diagnosed following completed chemoprophylaxis were treated as a result of a contact history and positive tuberculin test (with a Heaf conversion in one case). Both presented with non-specific symptoms (abdominal pain three years later and cervical lymphadenopathy 15 months later, respectively). In the first case there was a small speck of calcification on the chest radiograph and both cases had a positive 1 TU Mantoux test. Although neither had a further history of contact with tuberculosis both had further treatment.

If a diagnosis of tuberculosis is accepted in all four cases, the rates of subsequent tuberculosis were $5 \cdot 1$ and $5 \cdot 4$ per 1000 patient years for the treated and untreated groups respectively. The confidence interval for the difference in the rates is large $(-0.74$ to $+1 \cdot 28)$ because of the small numbers with subsequent tuberculosis.

\section{Discussion}

This study shows that treatment decisions based on the use of a 1 TU Mantoux test, rather than a Heaf test, in children with a history of neonatal BCG vaccination reduces the number of children receiving chemoprophylaxis. Although the numbers are small, there is no evidence from this study that this results in an appreciable increase in risk of subsequent disease.

Two children allocated to the no treatment group subsequently developed tuberculosis. This was due to failure to act on the results of the test rather than lack of diagnostic accuracy. 
By our own criteria these children should have received treatment. A further four children were not treated despite a positive Mantoux test but had no evidence of disease by the end of the study. The two children who were treated after a course of chemoprophylaxis represent a difficult problem. Their presenting symptoms were unconvincing and, in retrospect, undue emphasis was placed on a positive tuberculin test known to have been positive previously. Tuberculin sensitivity is generally longlasting with only $8.3 \%$ reversion per year even in the elderly population; ${ }^{6}$ recrudescence or repeat infection was unlikely to have caused their symptoms.

Initial studies of the Heaf test in non-immunised patients showed excellent correlation between Heaf grade 2 and a Mantoux 5 TU test with a cut off of $5 \mathrm{~mm}$ induration. ${ }^{7}$ Comparable studies have not been carried out after BCG vaccination. Guidelines in the USA make no allowances for previous immunisation ${ }^{8}$ but, as BCG vaccination is rarely used in that country, the magnitude of the problem is small. In a study of unselected day nursery children in the UK Crawshaw and Thomson' found two children in 100 were Heaf grade 3 at 2-3 years after neonatal BCG vaccination. Cookson and Cookson, ${ }^{10}$ reviewing the adolescent screening programme carried out prior to BCG immunisation, identified 760 ethnic Asians with Heaf grade 3 or more of whom three had evidence of pulmonary tuberculosis at the time and only one subsequently developed the disease. The implication of that study was that the chance of developing clinical disease was very low and that previous BCG vaccination might explain the strong Heaf reaction. The same may apply to contacts and recent immigrants, except that the chance of tuberculous infection is somewhat increased in these cases.

There is some evidence that repeat tuberculin testing may induce an immune reaction. ${ }^{11} 12$ In children who have previously been vaccinated with BCG this may result in a significant percentage showing a diagnostic conversion. ${ }^{12}$ In the present study the Mantoux tests were performed after the Heaf test and the predominant result was for the Mantoux test to be negative after a positive Heaf. About half of the Heaf converters subsequently had a negative Mantoux test result and this is an interesting group. It is unlikely that they can all be explained by poor technique in administering the Mantoux test. A true tuberculosis infection would be expected to give a positive Mantoux test result and the likely explanation for these cases is reinduction of BCG immunity by the first Heaf test which was not adequate to respond to the lesser inoculum dose of the 1 TU Mantoux test. None of this group was treated and none had evidence of subsequent disease.
The use of the Mantoux 1 TU test to distinguish the effect of tuberculosis infection from BCG vaccination has been investigated previously. ${ }^{13}$ These workers found that there was almost complete separation of the effects of BCG vaccination from cases of tuberculous disease using a $5 \mathrm{~mm}$ cut off after a $1 \mathrm{TU}$ Mantoux test, but only if the BCG was given in the neonatal period. The situation for contacts with no evidence of disease was not investigated.

For a study of this kind the follow up rate was excellent and it is unlikely that any disease was missed. Even the 10 patients who were untraceable had been followed in the clinic for at least six months and, although late cases can occur, disease is most likely to present early following contact. ${ }^{14}$

The decision to give chemoprophylaxis is based on the perceived risk to the individual child of developing disease. Although antituberculous chemotherapy in children is relatively safe, there are additional problems of clinic attendance, obtaining the medicine, supervision, and compliance. Use of the $1 \mathrm{TU}$ Mantoux test after neonatal BCG vaccination, in combination with a clinical assessment and radiography can reduce the number of children given chemoprophylaxis. A larger study would be needed to confirm that there is no real increase in risk of subsequent tuberculosis.

1 Comstock GW, Livesay VT, Woolpert SF. The prognosis of a positive tuberculin reaction in childhood and adolescence. Am $\mathcal{F}$ Epidemiol 1974;99:131-8.

2. De March-Ayuela. Choosing an appropriate criterion for true or false conversion in serial tuberculin testing. $\mathrm{Am}$ Rev Respir Dis 1990;141:815-20.

3 Kroger L, Katila ML, Korppi M, Brander E, Pietikainen $M$. Rapid decrease in tuberculin skin test reactivity at preschool age after newborn vaccination. Acta Pediatr preschool age after

4 Department of Health. Immunisation against infectious disease. London: HMSO, 1992.

5 Subcommittee of the Joint Tuberculosis Committee of the British Thoracic Society. Control and prevention of tuberculosis in Britain: an updated code. BMF 1990;300:995-9.

6 Perez-Stable EJ, Flaherty D, Schector G, Slutkin G, Hopewell PC. Conversion and reversion of tuberculin reaction in nursing home residents. Am Rev Respir Dis 1988;137:801-4.

7 Report to the Research Committee of the British Tuberculosis Association. A single tuberculin test for epidemiological use: comparison of four tests. Tubercle 1958; 39:76-83.

8 Joint statement of the American Thoracic Society and the Centers for Disease Control. Diagnostic standards and classification of tuberculosis. Am Rev Respir Dis 1990;142: 725-35.

9 Crawshaw PAG, Thomson AH. Heaf test results after neonatal BCG. Arch Dis Child 1988;63:1490-1.

10 Cookson JB, Cookson AGI. Does a positive Heaf test reaction in Asian schoolchildren predict later breakdown of tuberculosis? Thorax 1992;47:776-7.

11 Felten MK, Van Der Merwe CA. Random variation in tuberculin sensitivity in schoolchildren. Am Rev Respir Dis 1989;140:1001-6.

12 Friedland IR. The booster effect with repeat tuberculin testing in children and its relationship to BCG vaccination. $S A M T$ 1990;77:387-9.

13 Karalliedde S, Katugaha LP, Uragoda CG. Tuberculin response of Sri Lankan children after BCG vaccination at birth. Tubercle 1987;68:33-8.

14 Teale C, Cundall DB, Pearson SB. Time of development of tuberculosis in contacts. Respir Med 1991;85:475-7. 